The Astrophysical Journal, 252:133-146, 1982 January 1

(C) 1982. The American Astronomical Society. All rights reserved. Printed in U.S.A.

\title{
THE LATE-TYPE STELLAR CONTENT OF THE FORNAX AND SCULPTOR DWARF GALAXIES
}

\author{
Jay A. Frogel and V. M. Blanco \\ Cerro Tololo Inter-American Observatory ${ }^{1}$ \\ M. F. MCCARTHY ${ }^{2}$ \\ Specola Vaticana, Vatican City State \\ AND \\ JUDITH G. COHEN \\ Palomar Observatory, California Institute of Technology \\ Received 1981 March 16; accepted 1981 June 4
}

\begin{abstract}
A field of area 0.13 square degrees has been surveyed for late-type stars in each of the Fornax and Sculptor dwarf elliptical galaxies. $J H K$ photometric data have been obtained for most of the stars found. In Fornax, we have positively identified $25 \mathrm{C}$ stars and one $\mathrm{M}$ giant. In Sculptor, two relatively blue $\mathrm{C}$ stars and a small number of possible $\mathbf{M}$ giants have been identified. In contrast to the Magellanic Clouds, there are no M6-M9 giants in Fornax or Sculptor.

The mean value and the variance of the bolometric luminosity function for the Fornax $\mathrm{C}$ stars are $-4.66 \pm 0.47$, quite similar to the values for the Magellanic Cloud C stars. The colors of the Fornax $\mathrm{C}$ stars overlap those of the $\mathrm{C}$ stars in the Magellanic Clouds but are bluer in the mean. The large dispersion in the color-magnitude diagram of the Fornax $\mathrm{C}$ stars is interpreted as arising from a significant spread in age and/or metallicity in the stellar population of Fornax.

The $\mathrm{C}$ stars found in Sculptor are quite similar in color and luminosity to the $\mathrm{C}$ stars in the globular cluster $\omega$ Centauri and are at the faint end of the luminosity distribution of $\mathrm{C}$ stars found in the Magellanic Clouds and Fornax.

The ratio of cool $\mathrm{C}$ stars to $\mathrm{M}$ stars, as determined from identical survey techniques, increases dramatically along the sequence Milky Way, LMC, SMC, and Fornax. This increase, together with systematic changes in the colors of the $\mathrm{C}$ and $\mathrm{M}$ stars, can be understood as arising from a systematic decrease in the mean metallicity of the galaxies on this sequence. The lack of concurrent significant changes in $M_{\text {bol }}$ (mean) or $M_{\text {bol }}$ (max) of the carbon stars may not be consistent with current theories of $\mathrm{C}$ star formation and evolution.

The new data for Sculptor, as well as those previously published, point to a stellar population, and possibly a star-formation history, qualitatively similar to that of $\omega$ Cen. A sharp discontinuity between Fornax and Sculptor in some of the properties which characterize the late-type stellar population of these two systems stands in contrast to a rather smooth gradation in the same properties for Fornax and galaxies more massive than it. We speculate that this discontinuity could have arisen if Sculptor were stripped of its gas component at a much earlier time than Fornax.
\end{abstract}

Subject headings: galaxies: individual — galaxies: stellar content — stars: abundances stars: carbon - stars: late-type — stars: luminosities

\section{INTRODUCTION}

Low-dispersion spectroscopic surveys in the nearinfrared of selected fields in the Magellanic Clouds have shown that the ratio of carbon to M-type giants (of types C, 2 and M6 and later) in these systems is far larger than that for the Galaxy. These ratios are 15 for the Small Magellanic Cloud (SMC), 2 for the Large Magellanic Cloud (LMC), and only 0.003 for the nuclear bulge of the Milky Way (Blanco, Blanco, and McCarthy (1978); Blanco, McCarthy, and Blanco (1980); Blanco and

\footnotetext{
${ }^{1}$ Cerro Tololo Inter-American Observatory is operated by AURA, Inc. under National Science Foundation contract no. AST 78-27879.

2 Visiting Astronomer, Cerro Tololo Inter-American Observatory.
}

McCarthy 1981, unpublished observations; see also Table 4). These authors have suggested that the ratio of $\mathrm{C}$ to $\mathbf{M}$ stars in a galaxy is related to the galaxy's mean metallicity. Stellar interior calculations (e.g., Sackmann, Smith, and Despain 1974; Renzini and Voli 1981; Iben 1981) are in qualitative agreement with this prediction.

The other nearby companions to the Milky Way are the dwarf ellipticals in Sculptor and Fornax. Colormagnitude diagrams for Sculptor (Hodge 1965; Kunkel and Demers 1977) show a giant branch similar to that of a moderately metal-poor globular cluster, but with essentially no blue horizontal branch stars and a large number of RR Lyrae variables (e.g., van Agt 1978). Norris and Bessell (1978) argue that the apparent width of the 
Sculptor giant branch is intrinsic, similar to that of $\omega$ Centauri, and arises from a spread in metallicity at least as great as is represented by the difference between M3 and M92.

Demers, Kunkel, and Hardy (1979) have found that Fornax also possesses a wide giant branch which they interpret as indicative of a range in abundance amongst the stars which, in turn, has arisen from enrichment of the interstellar medium in Fornax via successive generations of star formation. Danziger (1973) also inferred that there is a range of abundances in Fornax on the basis of observations of the integrated light of its globular clusters. Further work by Demers and Kunkel (1979, hereafter DK) revealed a large number of stars with $(B-V)>2.0$ in Fornax. They proposed that at least a few of these red stars are $C$ stars and inferred a degree of similarity in the stellar content of Fornax and that of clusters in the SMC. Aaronson and Mould (1980, hereafter AM) provided spectroscopic confirmation for the presence of $\mathrm{C}$ stars in Fornax and strengthened evidence for the suggestion by Demers, Kunkel, and Hardy that star formation in Fornax has passed through several generations.

The purpose of this paper is first to present the results of a search for cool C and M stars in Sculptor and Fornax which parallels Blanco, McCarthy, and Blanco's (1980, herafter BMB) efforts in the Magellanic Clouds. With new infrared photometry for most of the candidate stars, our second objective is to give a quantitative comparison with a similar infrared study (Cohen et al. 1981, hereafter CFPE) of the Magellanic Cloud stars found by BMB.

\section{THE SURVEYS}

The low-dispersion spectroscopic surveys of the Fornax and Sculptor systems were carried out at the prime focus of the Cerro Tololo Inter-American Observatory (CTIO) $4 \mathrm{~m}$ reflector by V. M. B. and M. F. M. in a manner similar to the surveys of the Magellanic Clouds; the reader is referred to $\S$ II of BMB for details. Only one plate of each galaxy was obtained; plate centers and exposure times are given in Table 1.V.M. B. and M.F.M. then scanned each plate, independently, selecting and classifying candidate $\mathbf{C}$ and $\mathbf{M}$ stars. There were few disagreements, and these were generally confined to the candidates that were considered uncertain for other reasons, as discussed below.

Selection and classification of late-type stars in Fornax

TABLE 1

4 Meter Grism Plates a of Sculptor and Fornax

\begin{tabular}{cccc}
\hline \hline Galaxy & R.A. (1975) & Decl. (1975) & $\begin{array}{c}\text { Exp. Time } \\
\text { (minutes) }\end{array}$ \\
\hline Sculptor $\ldots \ldots \ldots \ldots \ldots$ & $00^{\mathrm{h}} 58^{\mathrm{m}} \cdot 6$ & $-33^{\circ} 47^{\prime}$ & 100 \\
Fornax $\ldots \ldots \ldots \ldots \ldots \ldots$ & 0238.5 & -3435 & 90 \\
\hline
\end{tabular}

${ }^{a}$ Kodak IV-N plates were hypersensitized with $\mathrm{AgNO}_{3}$ and exposed through a Schott RG 695 filter. The area of each circular field (diameter $23^{\prime}$ ) is 0.13 square degrees centered on the positions indicated. were relatively straightforward. The 34 stars with $\mathbf{M}$ or $\mathrm{C}$ classifications are listed in Table 2, where the first column gives a running number we have assigned to each star, the second column gives the star number from DK's list of red stars, and the third column gives our classification. Uncertainties for the $\mathbf{M}$ stars generally arise because of the difficulty in classifying a star of early spectral type from a grism plate alone. Figure 1 reproduces an area from a Palomar Observatory Sky Survey print with the stars from the first column of Table 2 identified.

Of the 34 cool stars found in Fornax, 25 can be classified unambiguously as $\mathrm{C}$ stars. The luminosity classification of the $\mathbf{M}$ stars, i.e., whether they are giants in Fornax or foreground galactic dwarfs, is considered in $\S$ III. The faintest Fornax stars classified as $\mathbf{C}$ or $\mathbf{M}$ and listed in Table 2 are about one magnitude brighter than our limit for classification. Thus, it is unlikely that we are missing late-type stars unless there is a population of them whose brightest members are significantly fainter than the faintest members of the population we have identified. Furthermore, the relatively blue $\mathrm{C}$ stars which we have found in Sculptor (see below) would, if present in Fornax, have been identified easily on our plate of the latter galaxy.

We have compared our list with that of DK. Thirty stars selected by them as being red were off of our grism plate. Nineteen stars are common to both lists, as noted in Table 2. Of these, we are uncertain of the indentifications for the four which are shown with colons after the DK numbers. Fourteen red stars from DK's list in the area of the grism plate were not selected by us as being $C$ or $\mathbf{M}$, could not be classified because of blending with another image on the grism plate, or were too close $(\ll 1$ mag) to the plate limit to be classified by us. Finally, 15 stars, nearly half our list, eight of which are C-type stars, were not selected out by DK on the basis of redness.

Even though Sculptor is at only half the distance of Fornax, the identification of late-type stars was made difficult by their apparent faintness. Of the ones found, few could be classified unambiguously. The first column of Table 3 lists a running number for the candidate stars identified in Figure 2, which is from a $4 \mathrm{~m}$ prime focus direct plate kindly taken for us by F. Schweizer. Many of these stars have photographic photometry by Hodge (1965), and his numbers are given in the second column of Table 3. Two of the stars in our list have photometry available from Kunkel and Demers (1977); they are denoted by KD numbers in the notes column. Finally, two large-amplitude red variables pointed out by van Agt (1978) are also indicated in the notes column. One of these is not in the field of the grism plate but is included in Table 3 since infrared data were obtained for it.

Only two of the cool stars found on the grism survey plate (nos. 4 and 12) can be identified unambiguously as $C$ stars in Sculptor. Both are considerably bluer than any we have found either in Fornax or in the Magellanic Clouds. This blueness is evidenced by long blue "tails" on the unwidened grism spectra which are bright enough that they would have been noticed if they had been present on any other $\mathrm{C}$ star image. 
TABLE 2

Cool Stars in the Field of the Fornax Dwarf Galaxy

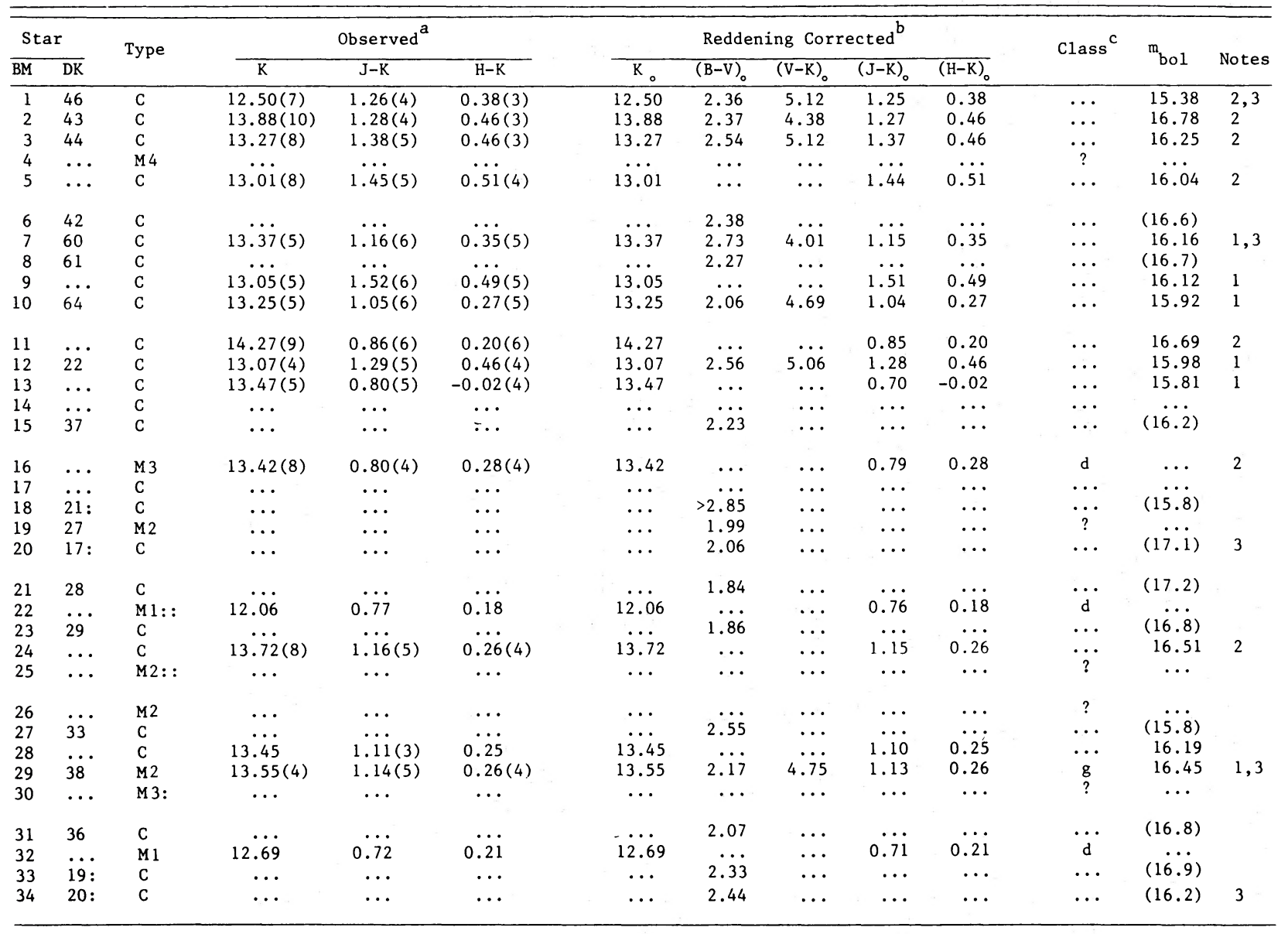

Notes.-(1) Observed at Las Campanas. (2) The large uncertainties in the K-magnitudes for these stars arose from an instrumental problem which had only minimal effect on the colors. (3) At least for these stars, the variability $V$ appears to be $\gtrsim 0.3$ mag (Demers and Kunkel 1979).

a The numbers in parentheses are uncertainties in units of hundredths of a magnitude when greater than 2.

b The $B V$ photometry is from Demers and Kunkel (1979). A reddening value of $E(B-V)=0.02$ was used.

c A luminosity classification is indicated only for noncarbon stars with infrared photometry as discussed in the text.

d For stars with infrared photometry, the bolometric magnitudes are derived from $K$-magnitudes and $(J-K)$ colors. For stars with optical data only, the $m_{\mathrm{bol}}$ are derived from these latter data and enclosed in parentheses. No values are given for the $\mathrm{M}$-type stars classified as foreground dwarfs.

The three stars listed as $\mathrm{C}:$ : are not faint but show absorption features in the region of the CN $\lambda \lambda 7945,8125$, and 8320 bands that are weak, and, therefore, the classification is rather uncertain. We emphasize that the two stars positively classified as $\mathrm{C}$ stars are spectroscopically quite distinct from these uncertain cases.

Those stars with an $\mathbf{M}$ followed by a spectral subclass in the third column of Table 3 could be classified unambiguously. Whether they are foreground galactic dwarfs or giants in Sculptor is discussed later. Stars with an M followed by one or two colons could be late $\mathrm{K}$ stars instead of M's. Stars with a question mark give the appearance, from their continua, of being cool, but the weakness of the absorption features makes more definitive classification impossible. These uncertain cases have been included in Table 3 because the survey of the Sculptor system yielded appreciably fewer red stars than the one of the Fornax system.

Finally, we point out that, in neither Sculptor nor Fornax, have we found any M stars as late as the M6-M9 ones identified in the Magellanic Clouds or "Baade's window" region of the galactic bulge (BMB; Blanco, Blanco, and McCarthy 1978; Blanco, Hoag, and McCarthy 1978).

\section{INFRARED OBSERVATIONS AND BOLOMETRIC CORRECTIONS}

Broad-band $J H K$ photometry was obtained for all but one of the Sculptor stars and 16 of the 34 Fornax stars in an identical manner and often on the same nights as 


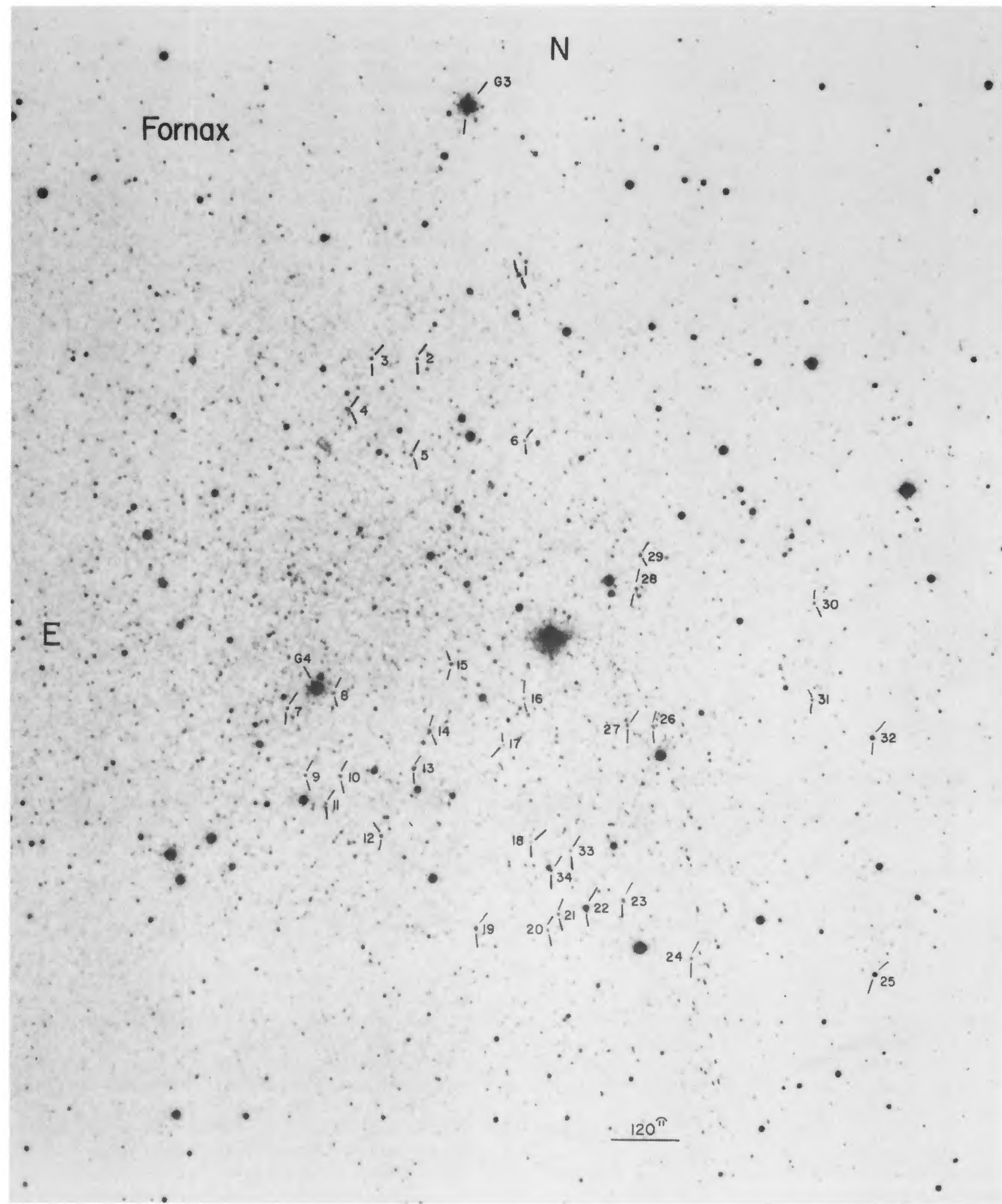

Fig. 1.-The Fornax galaxy as it appears on the Palomar Observatory Sky Survey; identified are all of the candidate late-type stars with numbering as in the first column of Table 2. Globular clusters 3 and 4 are also indicated. The approximate position of star 1 is R. A. $(1950) 02^{\mathrm{h}} 37^{\mathrm{m}} 36^{\mathrm{s}}$, decl. $(1950)$ $-34^{\circ} 32^{\prime} 51^{\prime \prime}$. 


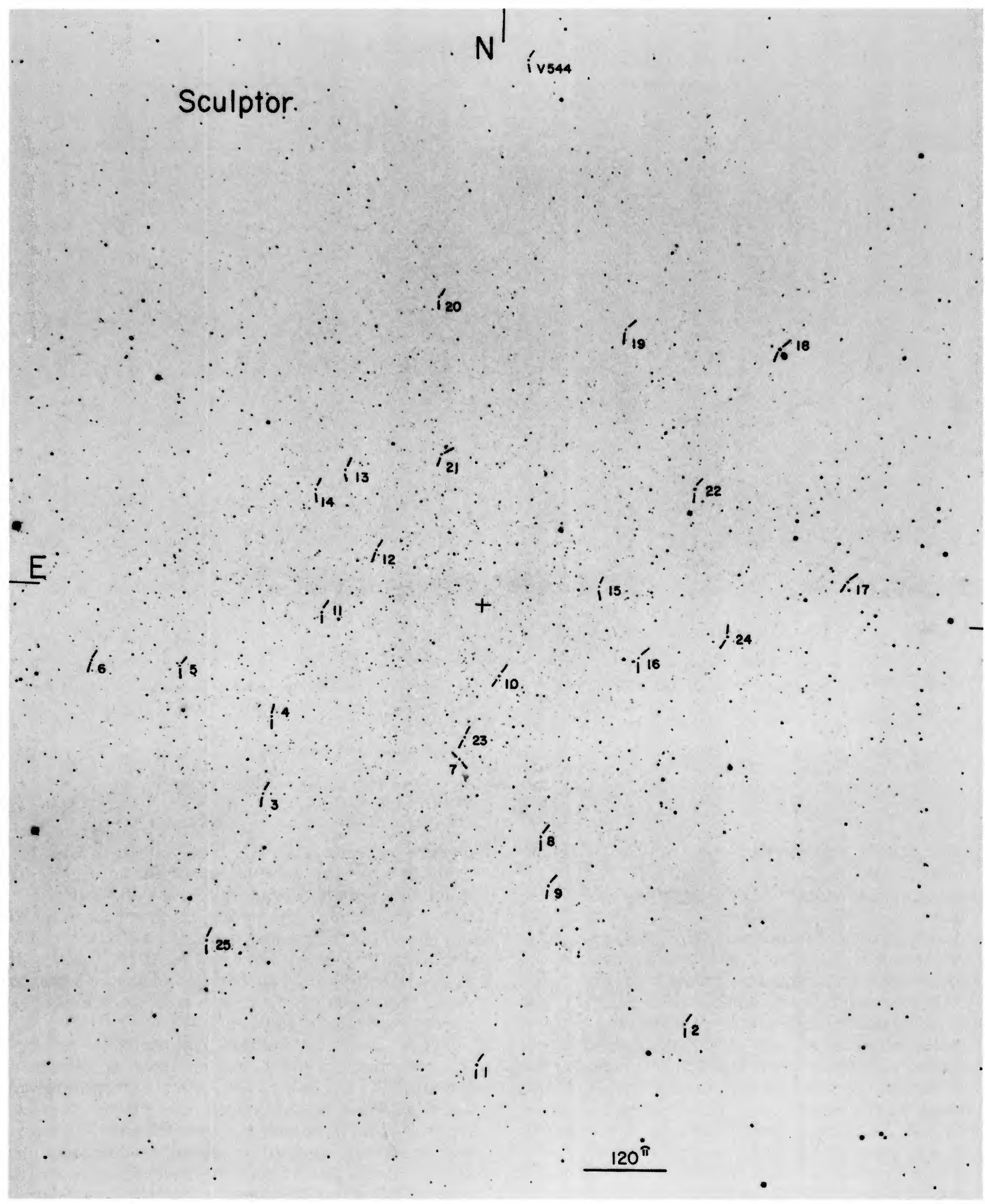

FIG. 2.-This print, made from a CTIO $4 \mathrm{~m}$ prime focus plate, identifies all of the candidate late-type stars found in Sculptor with numbering as in Table 3. The indicated scale is a mean value only since the outer portions of $4 \mathrm{~m}$ direct plates can be distorted by as much as $20^{\prime \prime}$ with respect to the plate center. The small "plus" near the center is located approximately at R. A. (1950) $00^{\mathrm{h}} 57^{\mathrm{m}} 38^{\mathrm{s}} 4$, decl. $(1950)-33^{\circ} 59^{\prime} 23^{\prime}$. Unfortunately, there are some plate and printing flaws which could confuse identification. The most obvious ones, which appear to be bright stars, are below and to the right of star 18; below and to the left of star 16; and to the left and somewhat below star 24 . 
TABLE 3

Cool Stars in the Field of the Sculptor Dwarf Galaxy

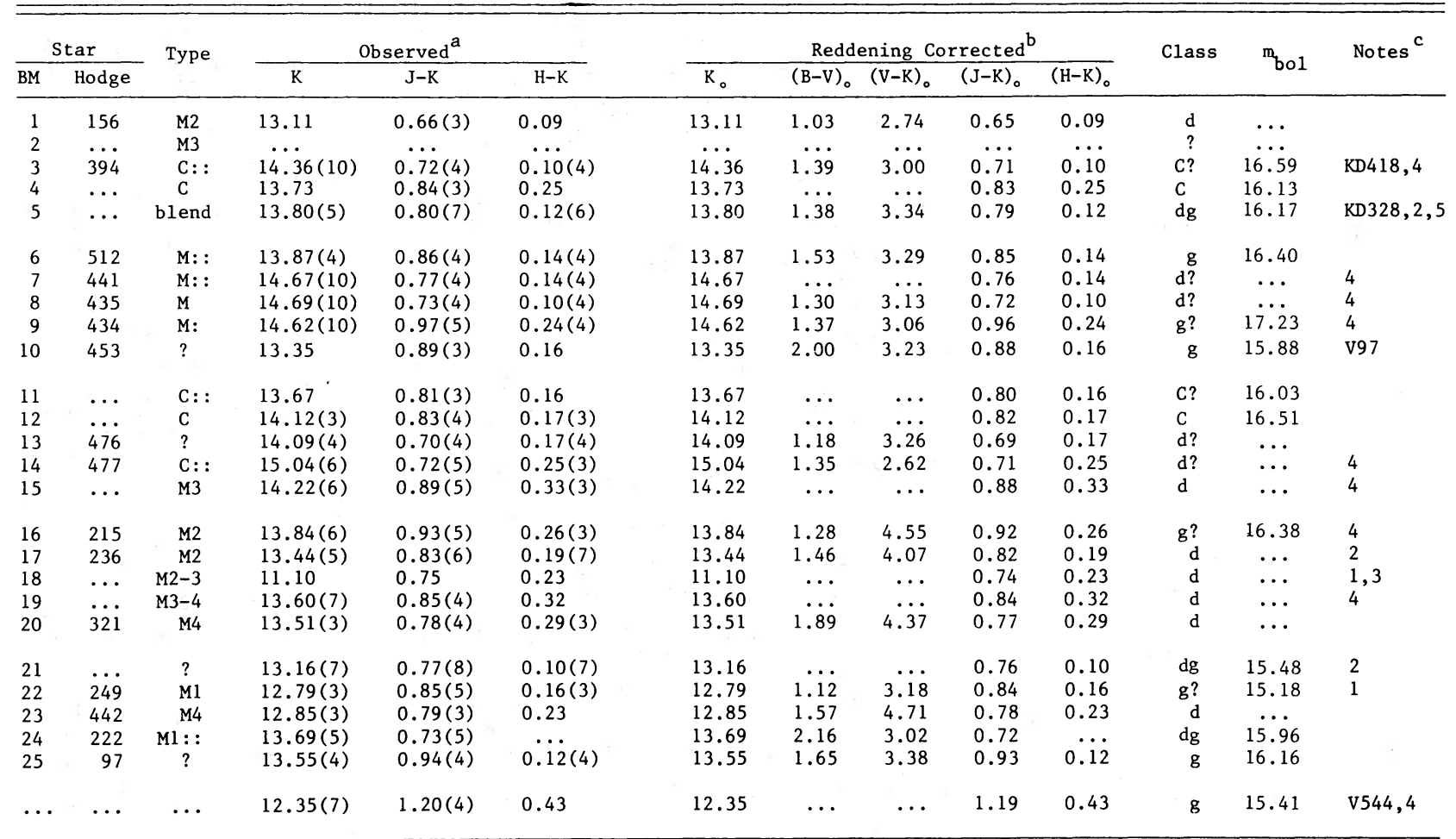

Notes.-(1) Observed at Las Campanas and CTIO. (2) Observed at Las Campanas. (3) Observed values of molecular absorption indices are $\mathrm{H}_{2} \mathrm{O}=0.165$ (3), $\mathrm{CO}=0.01$ (3). (4) The large uncertainties in the $K$-magnitudes for these stars arose from an instrumental problem which had only minimal effect on the colors. (5) Photographic $B V$ photometry from Kunkel and Demers 1977.

a The numbers in parentheses are uncertainties in units of hundredths of a magnitude when greater than 2.

b The $B V$ photometry is from Hodge 1965 , transformed as described in the text.

c Two stars which are identified by Kunkel and Demers 1977 are indicated. V97 and V544 are variables noted by van Agt 1978.

similar data for field and cluster stars in the Magellanic Clouds (CFPE; Frogel and Cohen 1981). Most of the observations were made with the CTIO $4 \mathrm{~m}$ reflector. The remainder were made on the $2.5 \mathrm{~m}$ du Pont reflector at Las Campanas. The observed $J H K$ colors and magnitudes are given in the fourth, fifth, and sixth columns of Tables 2 and 3 . These values are on the same photometric system as our previously published Magellanic Cloud and globular cluster observations. Systematic differences between infrared colors and magnitudes obtained at the two observatories have been found to be $0.01 \mathrm{mag}$ or less with dispersions consistent with the quoted observational uncertainties. A few stars have been observed in common with AM. The dispersion between their measurements and ours are 2-3 times the quoted uncertainties.

The $B V$ photometry for Fornax was taken from DK. We chose to use $E(B-V)=0.02$ for Fornax in view of its galactic latitude and the reddening of other objects in the vicinity, but we regard this as uncertain by \pm 0.02 . Corrected colors and magnitudes for Fornax are given in the seventh through eleventh columns of Table 2.

The $B V$ photometry for Sculptor is from Hodge (1965) unless noted otherwise. His $V$-magnitudes were made equivalent to those of Kunkel and Demers (1977) by using the mean difference quoted by the latter. The $(B-V)$ colors of Hodge were transformed according to the formula of Norris and Bessel (1978). Based on the discussion of Kunkel and Demers (1977), a value of $E(B-V)=0.02$ for Sculptor was adopted. Corrected colors and magnitudes are given in the seventh through eleventh columns of Table 3 .

For the C stars with infrared photometry, the bolometric corrections to the $K_{0}$-magnitudes were calculated from the $(J-K)_{0}$ colors via the mean relationship given in Frogel, Persson, and Cohen (1980). For the $\mathbf{M}$ giants (see $\S$ IV), the relationship between $\mathrm{BC}_{K}$ and $(J-K)_{0}$ in that paper was modified somewhat. Undoubtedly, the stars in Fornax and Sculptor are metal poor compared to galactic field stars. Since the $(J-K)_{0},(V-K)_{0}$ relation for metal-poor, noncarbon stars, as defined by globular cluster giants, differs somewhat from that for field giants (Frogel, Persson, and Cohen 1981), the former relationship based on Figure 13 of that paper (1981) was used to convert their Figures 10 and 11 so that $\mathrm{BC}_{K}$ could be 
derived from $(J-K)_{0}$ for the Fornax and Sculptor stars. The differences in the $\mathrm{BC}_{K}$ values are, in any case, small. As a consistency check on the procedure, $\mathrm{BC}_{K}$ was computed for the stars in Table 2 and 3 from $(V-K)_{0}$ colors as well. The mean differences and dispersions for seven stars in Fornax and nine in Sculptor are in the sense that $\mathrm{BC}_{K}$ derived from $(V-K)_{0}$ colors are $0.03 \pm 0.10$ and $0.04 \pm 0.15 \mathrm{mag}$ smaller, respectively, than those derived from $(J-K)_{0}$ colors. Separating the 16 stars into $C$ and $M$ types leads to the same result, namely that there are no significant differences in the $\mathrm{BC}_{K}$ values derived from the two colors. Dispersion in the two sets of values is consistent with the errors in the photographic $V$ photometry. Final values of apparent bolometric magnitudes derived from the infrared colors are given in the thirteenth columns of Tables 2 and 3 .

In order to examine the luminosity function of an unbiased sample of the Fornax C stars $(\S \mathrm{V})$, we need bolometric corrections for those for which we do not have infrared photometry. All but two of these stars have $(B-V)_{0}$ from Demers and Kunkel (1979). Thus, it was possible, by use of mean relationships between $(B-V)_{0}$, $(V-K)_{0}$, and $(J-K)_{0}$ derived from these data and from Mendoza and Johnson (1965) to estimate bolometric corrections to the $V$-magnitudes for the stars in Table 2 that have only $B V$ photometry. The resulting bolometric magnitudes are enclosed in parentheses in the thirteenth column of Table 2 . We estimate the uncertainties in these values as $0.2 \mathrm{mag}$ greater than in the values derived from $(J-K)_{0}$ which, for most cases, should be less than 0.1 mag.

\section{LUMINOSITY AND CLASSIFICATION OF THE STARS}

The possible and probable $\mathbf{M}$ stars found in Fornax and Sculptor have considerably fainter apparent magnitudes than the $\mathbf{M}$ stars, identified in the Magellanic Clouds by BMB. Therefore, we must consider the possibility that some of them are foreground dwarfs since, with the resolution and spectral region employed in the search, dwarfs and giants are indistinguishable.

We have used several, not necessarily independent, criteria to attempt to distinguish between late-type dwarfs and giants and to classify the stars with a question mark in the third column of Table 3. These criteria are based on the small differences in spectral energy distributions of dwarfs and giants of the same spectral subclass (Johnson 1966; Frogel et al. 1978). Figure 3 shows the mean $\left[(V-J)_{0},(J-H)_{0}\right]$ relation for galactic dwarfs and giants from Frogel et al. Stars from the two galaxies are identified individually by an $\mathrm{F}$ or an $\mathrm{S}$ followed by the number of the first columns of Tables 2 and 3. Three additional Fornax stars with $J H K$ photometry from AM are also identified on Figure 3 but with numbers from Demers and Kunkel (1979). Figure 4 is similar to Figure 3 except that it shows the $\left[(J-H)_{0},(H-K)_{0}\right]$ colors for the stars.

\section{a) The Fornax Stars}

All of the carbon stars identified in our survey field in Fornax have been classified unambiguously. None of these have anomalous colors when compared with other

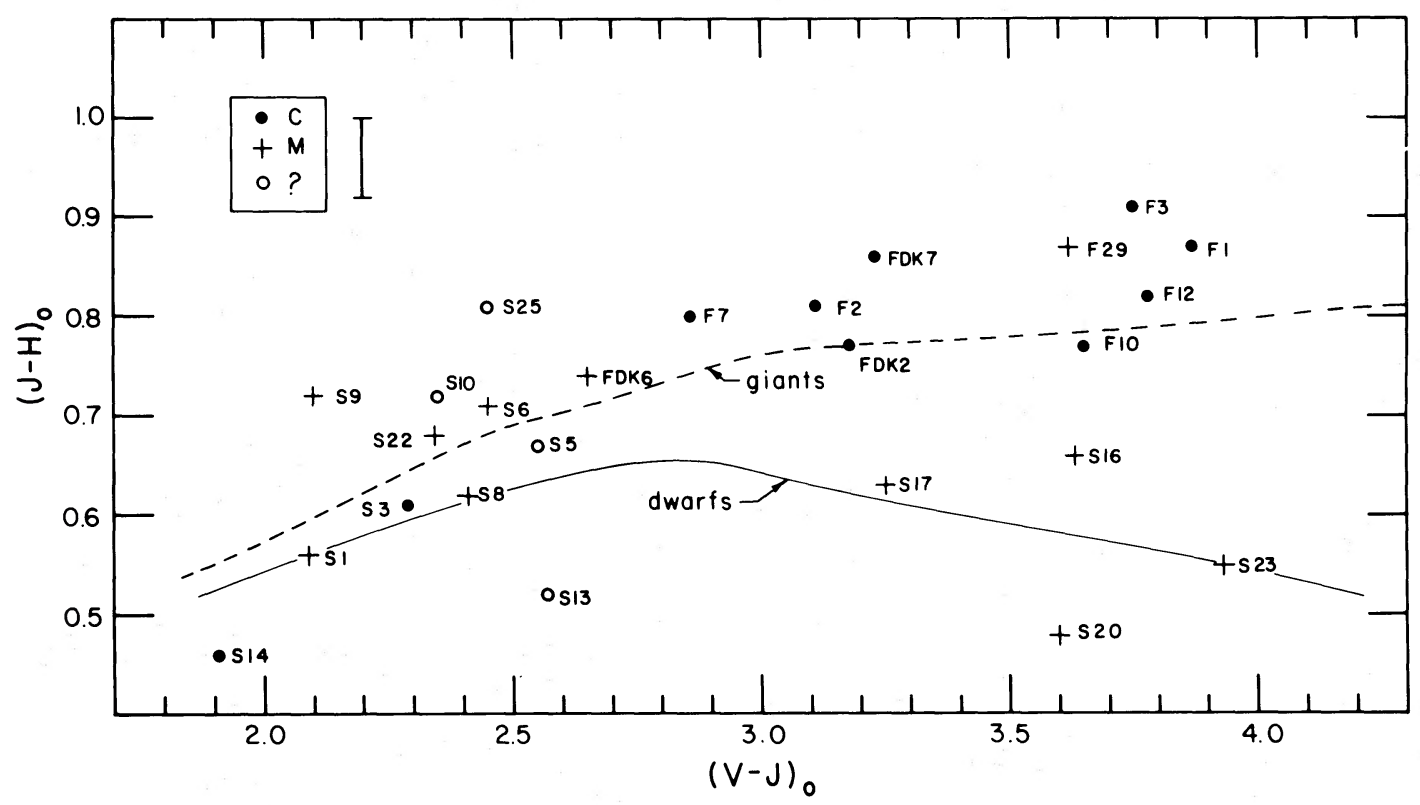

Fig. 3.-A color-color plot for Fornax $(\mathrm{F})$ and Sculptor (S) stars with infrared and $V$ data from Tables 2 and 3. For three of the Fornax stars (FDK), the infrared data are from Aaronson and Mould (1980). The lines show the mean relationship for galactic $\mathrm{K}$ and $\mathrm{M}$ dwarfs and giants (Frogel et al. 1978). The notations $C(O), \mathbf{M}(+)$, and ? (O) refer to the spectral classification in the third columns of Tables 2 and 3 . 


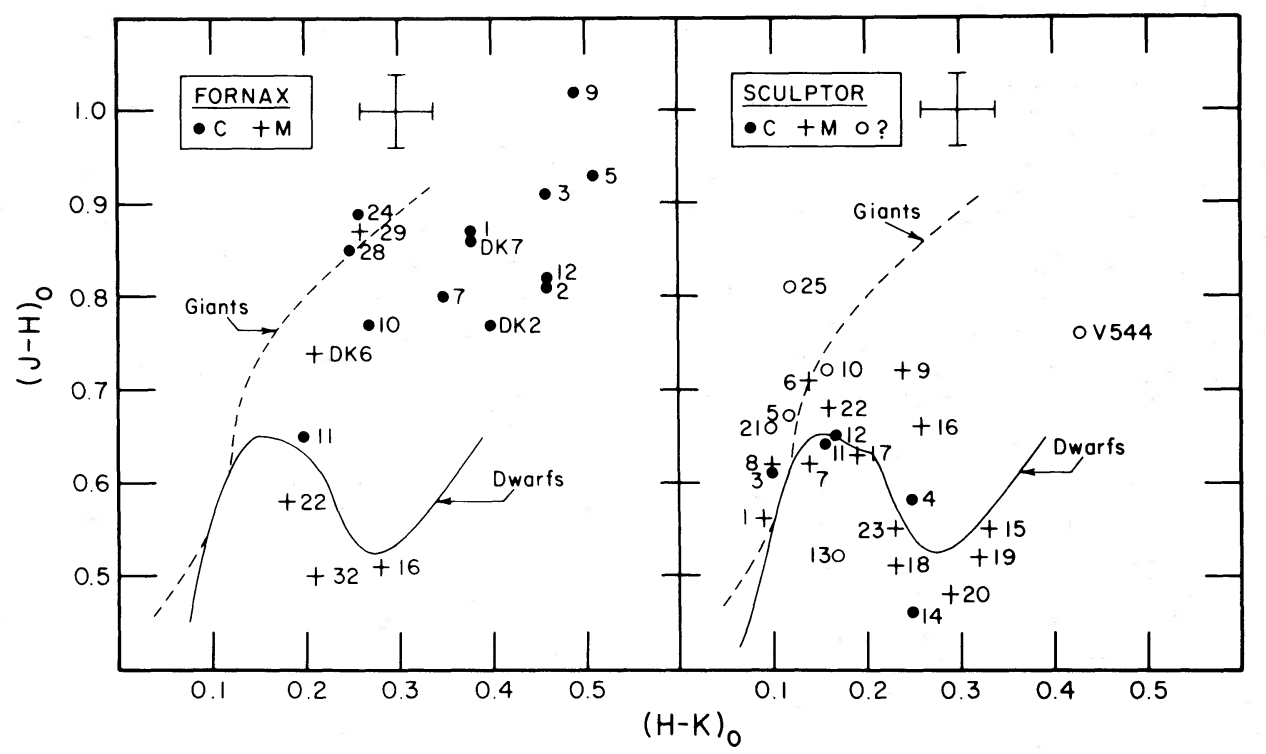

FIG. 4. - This figure is similar to Fig. 3 except for the coordinates chosen. The Fornax and Sculptor stars were separated for clarity.

C stars in the Magellanic Clouds (CFPE). The stars DK 2 and 7 have been classified spectroscopically by AM as $\mathrm{C}$ stars and have $J H K$ colors which are indistinguishable from the colors of survey stars.

The locations of Fornax stars 16, 22, and 32 in Figure 4 are consistent with their being dwarfs. Furthermore, their $(J-K)_{0}$ colors are probably too blue to be consistent with their being $\mathbf{M}$ giants but are consistent with an $\mathbf{M}$ dwarf classification. Star 29 from Table 2 has the colors expected of an M giant just as DK 6 probably does (Figs. 3 and 4$)$, although the red $(B-V)$ for star 29 is completely inconsistent with its $J H K$ colors given by AMregardless of its luminosity classification. (Perhaps it is a $\mathrm{C}$ star with exceedingly weak absorption features.) In any case, we shall consider DK 6 and star 29 as the only two probable $\mathbf{M}$ giants in Fornax with infrared photometry. ${ }^{3}$

\section{b) The Sculptor Stars}

Only two stars in Sculptor, nos. 4 and 12 in Table 3, have been unambiguously classified as $C$ stars. As we see below, their $J H K$ colors are consistent with those of a certain group of galactic $\mathrm{C}$ stars. The colors of stars 3 and 11 are completely ambiguous with regard to C-type or M-type or luminosity class. We shall consider them as "C?". Star 14 could well be an $M$ dwarf although its $(B-V)$ is a bit blue. It will not be considered further.

\footnotetext{
${ }^{3}$ Star 23 from Table 2 ( $\equiv$ DK 29) has been classified by us as $C$ while AM classify it as M1. The appearance of its near-infrared spectrum on our plates leaves little room for doubt that it is a C star. We suggest that the weakness of the features in the blue and visual spectral region (Fig. 1 of AM) could lead to a misclassification. The classification of nos. 1, 7, and 12 ( $\equiv$ DK 46, 60, and 22 respectively) as C stars agrees with AM's spectral classification. AM considered DK 33 ( $\equiv$ No. 27, Table 2) to be a $C$ star on the basis of its $J-H$ color. Our spectroscopic classification confirms this.
}

These classifications are given in the twelfth column of Table 3.

For the M's and the unknowns in Table 3, we looked for consistency between the $(V-K)_{0}$ and $(J-K)_{0}$ colors as functions of spectral type and between the locations of the stars in Figures 3 and 4. If general consistency between all criteria existed, then a $\mathrm{d}$ or $\mathrm{g}$ classification was assigned. If there were some inconsistency, a question mark was added. If there were complete ambiguity, usually because of lack of sufficient criteria, the star was listed as d/g. Star 10 (三V97) and V544 are classified unambiguously as g since dwarfs are not known to vary as these stars do. Stars in class $d$ or $d$ ? are not considered further.

\section{c) CLEANed Color-Color and Color-Magnitude Diagrams}

Figure 5 is a reddening corrected $\left[(\mathrm{J}-\mathrm{H})_{0},(\mathrm{H}-\mathrm{K})_{0}\right]$ plot for all stars from Tables 2 and 3 which have not been classed as $\mathrm{d}$ or $\mathrm{d}$ ?. Included in this figure are Fornax stars DK 2, 6, and 7 with infrared data from AM. Sculptor stars classed $\mathrm{g}$ ?, $\mathrm{d} / \mathrm{g}$, and the uncertain C's are plotted with parentheses. The mean relationships for galactic field C stars (CFPE) and M stars (Frogel et al. 1978) are indicated. Data for two C stars in $\omega$ Cen (nos. 55 and 70 , Persson et al. 1980) and for six C stars near the south galactic pole (Frogel and Phillips 1980, unpublished data) are also shown.

Figure 6 is a color-magnitude diagram for the Fornax and Sculptor stars in Figure 5. Distance moduli of $(m-M)_{0}=21.0$ and 19.47 were used for Fornax and Sculptor respectively. Fiducial, globular-cluster, giant branches for 47 Tuc, M3, and M92 are from Cohen, Frogel, and Persson (1978) and Frogel, Persson, and Cohen (1981). In addition to the $\omega$ Cen C stars, we also 


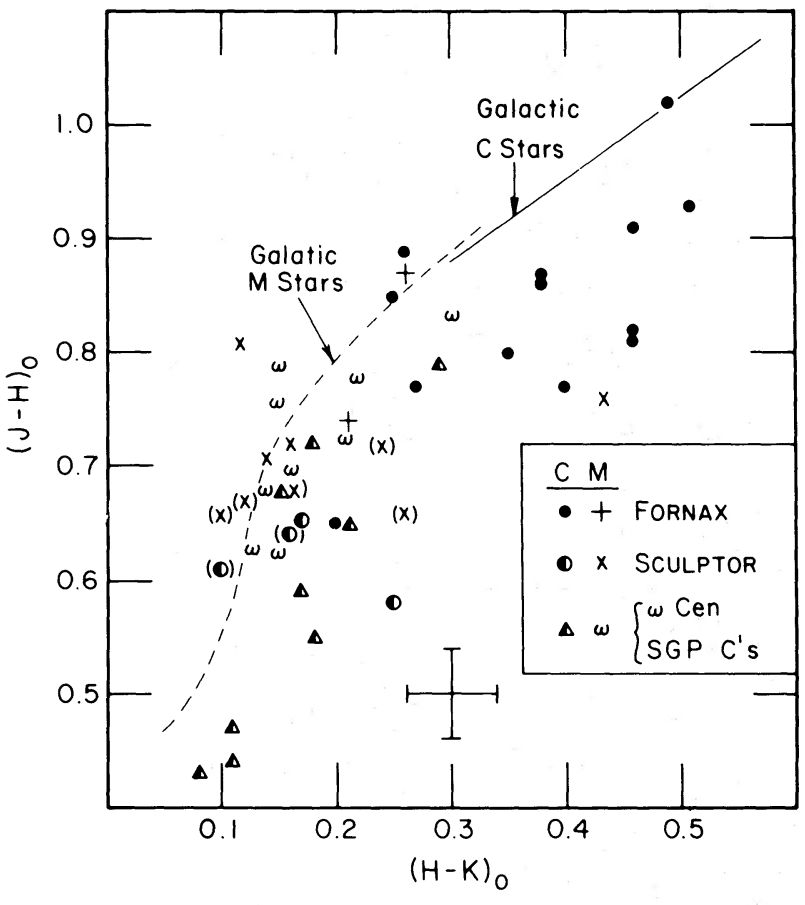

FIG. 5.-All stars from Tables 2 and 3 not classed as $d$ or $d$ ? are plotted. Symbols in parentheses are $\mathrm{g}$ ?, $\mathrm{d} / \mathrm{g}$, or C? from the twelfth column of Table 3. Included are DK 2, 6, and 7 from Aaronson and Mould (1980), two carbon stars and several brighter noncarbon stars from $\omega$ Cen (Persson et al. 1980), and 5 C stars located in the south galactic polar region (Frogel and Phillips 1980, unpublished data). The mean relationship for galactic $\mathrm{C}$ stars is from CFPE.

show the location of some of the oxygen-rich stars near the top of its giant branch (Persson et al. 1980), most of which are variables.

\section{THE STELLAR CONTENT OF FORNAX AND SCULPTOR \\ a) Fornax}

The Fornax dwarf elliptical possesses a late-type stellar population which consists almost entirely of $\mathrm{C}$ stars. There is a striking absence of late-type $\mathbf{M}$ stars. In this respect, at least, it is similar to the SMC (BMB; Blanco, Hoag, and McCarthy 1978), in agreement with the suggestions of DK and AM.

The Fornax $\mathrm{C}$ stars, at a given $(H-K)_{0}$, are significantly bluer in $(J-H)_{0}$ than the mean for galactic $\mathrm{C}$ stars (Fig. 5). This is the situation which obtains for the Magellanic Cloud C stars (CFPE), although the scatter in the Fornax sample does not permit us to say whether or not the displacement is as great as it is for the SMC. The reddest Fornax $\mathrm{C}$ stars are significantly bluer in all infrared colors than the reddest in the SMC which are, in turn, bluer than the reddest in the LMC. The same trend is true for the mean $(J-K)_{0}$ colors as well. At a given $(J-K)_{0}$, the Fornax $C$ stars display a considerable spread in magnitude (Fig. 6), as do the Magellanic Cloud field carbon stars (CFPE). In addition, for none of the samples is there an obvious dependence of bolometric magnitude on color.

Our spectroscopic survey of Fornax revealed only one (no. 29) M star which appears to be a bona fide member of that system on the basis of subsequent infrared photometry. One more has been identified by AM (but see note 3 above). The luminosities (about 1 mag greater than the tips of globular-cluster giant branches) and colors of these two stars are similar to the mean properties of the $M$ stars in the LMC (CFPE).

The bolometric luminosity function for the complete sample of Fornax C stars found in our survey is displayed in Figure 7, where we also show the luminosity function for a complete sample of SMC field C stars ${ }^{4}$ (CFPE). The mean and variance of the Fornax $\mathrm{C}$ star luminosity function are -4.66 and \pm 0.47 . Corresponding values for the SMC function are -4.84 and \pm 0.49 . We cannot say if the difference in the means is significant; that for Fornax is based on $(m-M)_{0}=21.0$ (Demers and Kunkel 1979), which is, in fact, at the low end of the range quoted by Hodge (1971), viz., $21.4(+0.4,-0.5)$.

${ }^{4}$ The luminosity functions in $M_{I}$ and $M_{\text {bol }}$ for the LMC and SMC are indistinguishable with regard to both the variance and the mean if the true distance modulus of the SMC is $0.5 \mathrm{mag}$ greater than that of the LMC (BMB; CFPE).

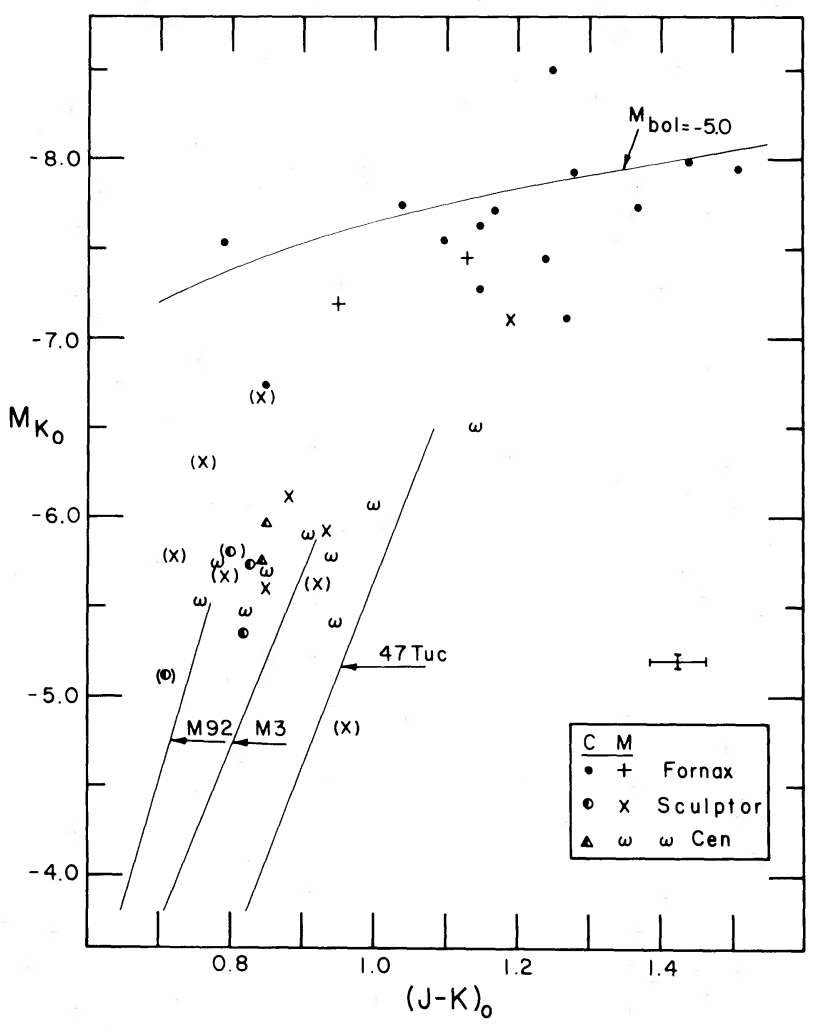

FIg. 6.-Colors and magnitudes for the stars in Fig. 5 except for the south galactic polar C-stars. The line of constant $M_{\text {bol }}$ is for C stars only and is from Frogel, Persson, and Cohen (1980). Fiducial giant branches for globular clusters are from Cohen, Frogel, and Persson (1978) and Frogel, Persson, and Cohen (1981). The values of $(m-M)_{0}=19.47$ and 21.0 are for Sculptor and Fornax respectively. 


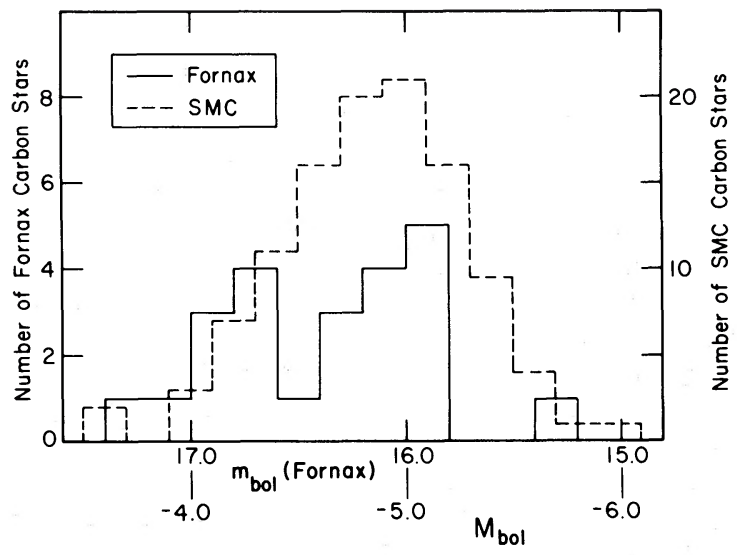

FIG. 7.-The luminosity function for the Fornax (solid line) C stars from Table 2 only. A value of $(m-M)_{0}=21.0$ was used. Bolometric magnitudes have been derived as discussed in the text. The luminosity function for the SMC (broken line) is from CFPE. A value of $(m-M)_{0}=19.1$ was used for the SMC

\section{b) Sculptor}

Although the distance modulus of Sculptor is $1.5 \mathrm{mag}$ less than that of Fornax, we have been able to detect only a handful of cool stars that we feel are probable members of this dwarf galaxy.

The two carbon stars that have been identified positively in Sculptor (nos. 4 and 12) are demonstrably different from essentially all of those found in similar surveys of the Magellanic Clouds (BMB) and Fornax in terms of blueness and faintness. However, they are quite similar in color and luminosity to the two C stars in $\omega$ Cen (Persson et al. 1980). Their colors are also similar to those of several supposed Population II C stars in the region of the south galactic pole (Frogel, and Phillips 1980, unpublished data). The two questionable C stars in Sculptor (nos. 3 and 11) are indistinguishable photometrically from the two confirmed ones. As we have noted elsewhere (Frogel et al. 1981), the $\omega$ Cen and Sculptor C stars have luminosities that lie at the lower limit of the luminosity functions for complete samples of both LMC and SMC C stars. In particular, neither the Sculptor nor the $\omega$ Cen C stars have luminosities that are significantly greater than the tips of galactic globular cluster giant branches.

If we exclude the red variable V544, then the three Sculptor stars which we have identified as the most probable candidates for $\mathbf{M}$ giants intermix with the brightest stars from $\omega$ Cen in color-color and colormagnitude plots (Figs. 5 and 6). The star V544 is distinctly redder and more luminous than these; however, its location in Figures 5 and 6 is quite close to the mean positions of the large-amplitude variables V1-V4 in 47 Tuc (Frogel, Persson, and Cohen 1981). ${ }^{5}$ At least one of

\footnotetext{
5 The explanation proposed previously to explain the unusually red $(H-K)_{0}$ colors of the 47 Tuc variables involves the effects of $\mathrm{H}_{2} \mathrm{O}$ absorption on the infrared energy distribution, particularly on the flux in the $H$ filter. Presumably, a similar explanation is applicable to V544, although confirmation of strong $\mathrm{H}_{2} \mathrm{O}$ absorption should be made.
}

the other three probable Sculptor $\mathbf{M}$ stars is a variable as well (no. $10 \equiv$ V97). It would be valuable to establish the luminosity of the $M$ stars we classify as $g$ ? and $d / g$ since, certainly, the two brighter and redder ones have few counterparts in galactic globular clusters but many in LMC clusters (Frogel and Cohen 1981).

\section{INTERPRETATION AND A COMPARISON WITH OTHER GALAXIES}

Reasonably complete surveys of the late-type stellar content of five galaxies now exist-the nuclear bulge region of the Milky Way (Blanco, Blanco, and McCarthy 1978), the Large and Small Magellanic Clouds (BMB), Fornax, and Sculptor. As summarized in Table 4, these five galaxies span most of the entire known range of galaxian mass and luminosity and, at the faint end, touch the upper end of the globular cluster distribution. Parameters which characterize the observed late-type stellar content of the five galaxies are also given in Table 4; they show a clear progression and, at the same time, considerable uniformity in the characteristics of the coolest stars in the galaxies. In the following discussion of Table 4, we assume that only two physical parameters are of primary importance - the mean age of these cool giants and the mean abundance of heavy metals which characterized the interstellar medium at the mean epoch of star formation. Many currently popular theories of star formation propose that these two parameters are intimately related to one another and that they may both be determined by another, more global parameter, i.e., the mass of the parent galaxy.

Furthermore, even though there are a number of apparently serious disagreements between theory and observation (CFPE; Richer 1981), we still assume that C star production results from helium-shell flashing in luminous giants with double-shell energy sources (e.g., Sackmann, Smith, and Despain 1974; Iben and Truran 1978; Renzini and Voli 1981) and that it is not yet necessary to invoke a fundamentally different physical mechanism (e.g., mixing via rotation) as has been suggested by Scalo and Miller (1979) to explain the origin of most of the $\mathrm{C}$ stars which are under discussion.

The most striking aspect of Table 4 is the sharp increase in the ratio of $\mathrm{C}$ stars to late $\mathrm{M}$ stars as one goes from the metal-rich to the metal-poor systems. BMB pointed this out in comparing the Magellanic Clouds to the Galaxy and suggested that the increase arose from differences in metallicity. A similar increase in the ratio of $C$ stars to $M$ stars is also strongly evident as one goes from the galactic center region to the galactic anticenter within the Milky Way (Blanco 1965). With the helium-shell flash mechanism operative in producing $C$ stars, one expects it to be easier to produce $\mathrm{C}$ stars out of metal-poor giants than out of metal-rich giants of similar mass because less carbon has to be mixed to the surface to get $\mathrm{C} / \mathrm{O}>1$ in the former stars. Furthermore, all other things being equal, we expect far fewer late $\mathbf{M}$ stars in metal-poor systems than in metal-rich systems simply because giant branch tracks are shifted to higher temperatures in the former, and asymptotic giant branch (AGB) evolution is 
TABLE 4

The late-Type Stellar Content of Five Galaxies

\begin{tabular}{|c|c|c|c|c|c|c|}
\hline \multirow[b]{2}{*}{ GALAXY } & \multirow[b]{2}{*}{$\log L / L_{\odot}$} & \multirow[b]{2}{*}{$\log M / M_{\odot}$} & \multirow[b]{2}{*}{$\mathrm{C} / \mathbf{M}^{\mathrm{a}}$} & \multicolumn{2}{|c|}{ C Stars } & \multirow{2}{*}{$\frac{\text { M STARS }}{M_{\text {bol }}(\max )}$} \\
\hline & & & & $(J-K)_{\max }^{\mathrm{b}}$ & $\bar{M}_{\text {bol }}{ }^{\mathrm{c}}$ & \\
\hline Milky Way ........ & $\sim 10$ & 11.1 & $<0.01^{\mathrm{k}}$ & $2.0^{\mathrm{f}}$ & $-4.8^{\mathrm{e}}$ & $-5.1^{\mathrm{h}, \mathrm{i}}$ \\
\hline LMC .............. & 9.3 & 10.0 & $2^{m}$ & $1.85^{\mathrm{f}}$ & $-4.85^{\mathrm{f}}$ & $-5.2^{\mathrm{f}}$ \\
\hline SMC $\ldots \ldots \ldots \ldots \ldots$ & 8.7 & 9.2 & $15^{\mathrm{n}}$ & $1.7^{\mathrm{f}}$ & $-4.84^{\mathrm{f}}$ & $\sim-5^{\mathrm{m}}$ \\
\hline Fornax $\ldots \ldots \ldots \ldots$ & $7.3^{\mathrm{d}}$ & $7.3^{\mathrm{d}}$ & $\geq 25^{\mathrm{j}}$ & $1.5^{\mathrm{j}}$ & $-4.66^{j}$ & $-4.4^{j}$ \\
\hline Sculptor .......... & $6.2^{\mathrm{d}}$ & $6.5^{\mathrm{d}}$ & ? & $0.85^{\mathrm{j}}$ & $-3.15^{\mathrm{j}}$ & $-3.3^{\mathrm{j}}$ \\
\hline$\omega$ Cen $\ldots \ldots \ldots \ldots \ldots$ & $6.1^{1}$ & $\sim 6$ & ? & $0.85^{8}$ & $-3.35^{\mathrm{g}}$ & $-3.4^{8}$ \\
\hline
\end{tabular}

a These are the ratios by number of stars of type C,2 and M6 and later as determined by essentially complete grism surveys. The upper limit for the Milky Way is based on new work on the solar neighborhood by Blanco 1981 (unpublished data). The nuclear bulge of the Milky Way surveyed by Blanco, Blanco, and McCarthy 1978 has an even lower upper limit since there is only one C star but 300 stars of M6 and later. The value for the SMC is based on a recently completed survey of many new fields in addition to the two contained in BMB. The total number of $\mathbf{C}$ and $\mathbf{M}$ stars in the surveyed fields is now over 600 . In view of the scarcity of late M's in the BMB survey of the SMC, their ratio of 27 is not significantly different from the new ratio of 10 . The number for Fornax is a lower limit since the one $\mathbf{M}$ star which is a giant in Table 2 is earlier than M6. The ratio for Sculptor and $\omega$ Cen is indeterminate since neither system has stars as late as M6.

${ }^{b}$ This is the color of the reddest $\mathrm{C}$ stars excluding the few long period variables that are markedly separated from the bulk of the stars (cf. CFPE). For the Milky Way, $(J-K) \max$ is for field C stars, e.g., CFPE 1980.

c For Sculptor and $\omega$ Cen, these are just the mean values for the two positively identified C stars in each system with infrared photometry from the sources noted. For the Milky Way, it is a mean value given by Baumert 1974 for a group of the field C stars which should be spectroscopically similar to those found in the grism surveys. For the LMC, SMC, and Fornax, the means are from the luminosity functions of unbiased samples of stars of type C,2 and later (see discussions in BMB and CFPE).

d Hodge 1971.

e Baumert 1974.

${ }^{\text {f }}$ Cohen et al. 1981.

g Persson et al. 1980.

${ }^{\text {h }}$ Frogel 1981.

${ }^{\text {i }}$ Frogel and Whitford 1981.

${ }^{j}$ This paper.

${ }^{k}$ Blanco, Blanco, and McCarthy 1978.

${ }^{1}$ Kron 1974.

m Blanco, McCarthy, and Blanco 1980.

${ }^{\mathrm{n}}$ Blanco and McCarthy 1981 (unpublished observations).

predicted to proceed at nearly constant temperature above the giant branch (GB) (e.g., Gingold 1974). Thus, not only will one get a higher fraction of oxygen-rich stars turning into carbon-rich stars, but there will be fewer late $\mathbf{M}$ giants to start with in a metal-poor system compared to a metal-rich system. Thus, we interpret the sequence of ratios of $\mathbf{C}$ to $\mathrm{M}$ stars as primarily reflecting a sequence of decreasing mean metallicity.

We have argued elsewhere (CFPE) that the $J-K$ colors of $\mathrm{C}$ stars may be determined mainly by the degree of enhancement of, and subsequent blanketing by, carbon molecules in a star's atmosphere and only secondarily by stellar temperature. Because of the relative importance of $\mathrm{CN}$ opacity, the $J H K$ colors of $\mathrm{C}$ stars will depend on the degree of blanketing and hence on metallicity via the abundance of nitrogen. Thus, the decreasing values of $(J-K)_{0}$ which characterize the red limits of the C star distributions are also what would be expected if the sequence of galaxies in Table 4 were one of decreasing mean metal abundance.

The luminosity function of the Fornax $\mathrm{C}$ stars is quite similar to those of the LMC and SMC with regard to its mean luminosity and dispersion. As discussed by CFPE, theoretical C star luminosity functions (e.g., Iben and Truran 1978; Iben 1981; Renzini and Voli 1981) differ in several respects from observed luminosity functions. The most obvious difference is the fact that the observed mean luminosities of $\mathrm{C}$ star populations are up to $2 \mathrm{mag}$ fainter than predicted means. (Several, albeit not completely satisfactory, ways out of this difficulty have been discussed by Wood 1981, Iben 1981, and Renzini 1981; but see CFPE and Frogel et al. 1981). Our new luminosity function for an unbiased sample of $\mathrm{C}$ stars from Fornax further emphasizes this disagreement.

A second difference between theoretical predictions and observations concerns the dependence of mean C star luminosity on metallicity. Given two stars of equal mass but different metallicities, theory leads us to expect that the metal-poor one will turn into a $\mathrm{C}$ star at lower luminosity than will the metal-rich one since, again, less carbon has to be mixed to the surface to get $\mathrm{C} / \mathrm{O}>1$ (e.g., Sackmann, Smith, and Despain 1974; Renzini and Voli 1981). Thus, given similar star-formation histories in two galactic systems (i.e., similar initial stellar mass functions 
and star-formation rates), the mean luminosity of C stars should be lower in the more metal-deficient system. This expectation does not seem to be fulfilled in the case of the Magellanic Clouds (BMB; CFPE), and there is only marginal evidence for it if the Fornax galaxy is compared to the Magellanic Clouds. Unfortunately, the expected effect (at least as large as 0.2 mag [Iben 1981]) could be contained entirely within the uncertainties in the relative distance moduli of the three systems.

In fact, it is surprising, that, although Fornax is so much less massive and luminous than the Magellanic Clouds, the $\mathrm{C}$ star luminosity functions of the three systems are so similar. The observed luminosity functions are expected to depend in a gross way on the stellar initial mass function and the stellar birthrate (Iben 1981; Renzini and Voli 1981; CFPE). In the Magellanic Clouds, there is ample evidence that star formation has occurred continuously over a time scale of the order of $10 \times 10^{9}$ years, and, in particular, that it has occurred up to a fairly recent epoch. The LMC and SMC both possess a welldefined, old stellar population in addition to early-type and late-type supergiants and $\mathrm{H}$ II regions. Fornax, on the other hand, has no $\mathrm{H}$ il regions, no detectable $21 \mathrm{~cm}$ emission, and apparently no detectable dust (see review by Hodge 1971). The star-formation rate for Fornax during the last $10^{9}$ years has, therefore, been lower than in the distant past so that, relative to a system with a more constant star-formation rate, one would expect the bright end of the $\mathrm{C}$ star luminosity function to be truncated at a lower luminosity than for the LMC and SMC. Carbon star luminosity functions for these galaxies (see Fig. 7) do not provide evidence supporting this prediction, although uncertainties in the distance moduli combined with the small total number of stars prevents us from drawing a definite conclusion.

A further interesting result may be obtained from a comparison of the number of $\mathrm{C}$ stars in each galaxy scaled by the galaxy's total luminosity. Blanco and McCarthy (1981, unpublished observations) have extended the BMB survey of the Magellanic Clouds and find that the total numbers of $C$ stars of type C, 2 and later in the LMC and in the SMC to be 14,000 and 3,000 respectively, with an uncertainty of \pm 500 in both cases. By comparing the area we have surveyed in Fornax with the area over which Demers and Kunkel (1979) find red stars, and with the extent of the galaxy on a blue plate taken with the $2.5 \mathrm{~m} \mathrm{du}$ Pont telescope at Las Campanas by Alan Dressler, we estimate that the total number of $\mathrm{C}$ stars in Fornax is $2-4$ times the number we have found, i.e., we estimate that there are 50-100 C stars in Fornax. Also, to within the large uncertainties of limited statistics, the distribution of these $\mathrm{C}$ stars is qualitatively similar to the overall light distribution in Fornax. Referring to the luminosities in Table 4, we conclude that, to within the stated uncertainties, the numbers of $C$ stars in the $L M C$, the SMC, and Fornax, is proportional to the galaxies luminosities. This fact, together with the other similarities we have already pointed out between the $\mathrm{C}$ stars in these three systems, leads us to suggest a similar age and evolutionary history for the $\mathrm{C}$ stars in the three galaxies.
If the Fornax $\mathrm{C}$ stars came from a population with a small range in age and metallicity, then one might expect them to define a $\left[K_{0},(J-K)_{0}\right]$ relationship with little scatter since their precursors would then be oxygen-rich giants which themselves would define an essentially dispersonless giant branch (Frogel and Cohen 1981). Thus, we interpret the large dispersions exhibited by the Fornax, SMC, and LMC C stars in color-magnitude diagrams such as Figure 6 here and Figure 7 of CFPE as arising from the significant spread in age and/or metallicity of the stellar populations in Fornax and the Magellanic Clouds. That such a spread exists in the Magellanic Clouds is generally accepted. Evidence for it in Fornax comes from the observations of van den Bergh (1969), Danziger (1973), and Demers, Kunkel, and Hardy (1979). Also, as has been emphasized by Mould and Aaronson (1980) and AM, the fact that a stellar population has C stars of luminosity comparable to the ones in Fornax and the Magellanic Clouds, can be taken as prima facie evidence that a substantial fraction of that population must have an age significantly younger than the ages of galactic globular clusters. We tend not to favor the possibility that the $\mathrm{C}$ stars in Fornax are old, i.e., $\gtrsim 10^{10}$ years, and the result of binary evolution because of the similarities that exist between the $\mathrm{C}$ stars in Fornax and the Magellanic Clouds. The C stars in the Clouds appear to be associated with an intermediate-age population (CFPE; Frogel and Cohen 1981; Mould and Aaronson 1980, and references therein). Furthermore, whatever the precursors of Fornax $\mathrm{C}$ stars are, they come from a population which apparently is spread out through the entire Fornax system.

Ignoring differences that might result from metallicitydependent, mass loss rates and statistical effects due to small sample sizes, the core mass of a giant ascending the asymptotic giant branch will be greater in systems of high metallicity at a given age because of slower evolutionary rates, and in young systems at a given metallicity (e.g., Mould and Aaronson 1980; Frogel 1981) because of greater initial mass. The luminosity of an AGB star is directly proportional to its core mass via a relationship such as that discussed by Paczynski (1971). Thus, the relatively faint maximum luminosities reached by the $\mathbf{M}$ stars in Fornax and Sculptor can be understood as arising from a combination of these systems' lower metallicities and their probably greater age for the mean starformation epoch, as compared to the Magellanic Clouds. The fact that $M_{\text {bol }}(\max )$ for the $M$ stars in the nuclear bulge of the Milky Way (Frogel 1981; Frogel and Whitford 1981) is not any greater than that for the Magellanic Clouds, even though the galactic bulge $\mathbf{M}$ giants undoubtedly have a considerably higher mean metallicity than those in the Clouds (e.g., Whitford and Rich 1981) is due, we suggest, to a sufficiently greater mean age for the bulge stars. In fact, a characteristic age for the galactic bulge should be comparable to that for the giant elliptical galaxies, given their similar stellar populations (Whitford 1978). Late-type $\mathbf{M}$ giants drawn from the relatively younger disk population of the Milky Way should have considerably higher bolometric luminosities than the bulge giants. 
Let us turn our attention now to the Sculptor system. As alluded to above, the presence of $\mathrm{C}$ or $\mathbf{M}$ stars much brighter than the luminosity of the helium-core flash in a stellar population can be taken as evidence that at least part of that population has to be of an age significantly (i.e., more than $2-3 \times 10^{9} \mathrm{yr}$ ) younger than that usually associated with galactic globular clusters (e.g., Catchpole and Feast 1973; Feast and Lloyd-Evans 1973; Mould and Aaronson 1980; AM). These arguments are based on current theoretical beliefs concerning mass loss rates, giant branch evolution, and luminosity-core-mass relations for asymptotic giant branch stars. The only reasonable certain, luminous $M$ star in Sculptor is V544. However, its luminosity is not greater than the luminosities of four large-amplitude red variables in 47 Tuc. The presence of these luminous variables is not generally taken as evidence that 47 Tuc is significantly younger than more metal-poor globulars, and, in fact, with the lower metallicity of 47 Tuc proposed by Pilachowski, Canterna, and Wallerstein (1980), its color-magnitude diagram is consistent with an age similar to that of the more metal-pore clusters. By analogy, then, Sculptor need not have a component to its stellar population which is significantly younger than its oldest component, exemplified by its large population of RR Lyrae variables.

What about the blue C stars we have found in Sculptor? Conceivably, they could represent the faint end of the $\mathrm{C}$ star luminosity functions we have found to be common to the Magellanic Clouds and Fornax, all of the brighter C stars in Sculptor having died with no recent star formation to replace them. However, we tend to favor the suggestion put forward by Scalo and Miller (1979) who maintain that $\mathrm{C}$ stars with luminosities comparable to the tips of galactic globular clusters (e.g., the ones in $\omega$ Cen and in Sculptor) are formed by some process distinct from that which is responsible for the more luminous objects. In fact, our rather limited Sculptor data as shown in Figures 5 and 6 are quite consistent with the analogy Norris and Bessell (1978) drew between Sculptor and $\omega$ Cen. The fact that $\omega$ Cen has a primarily blue horizontal branch while Sculptor's is primarily red could merely reflect a small (e.g., $1-2 \times 10^{9} \mathrm{yr}$ ) age difference (Castellani, Giannone, and Renzini 1973; Kunkel and Demers 1977)

Finally, we ask why it is that even though Fornax and Sculptor differ in mass by only about a factor of 10 , their late-type stellar populations are markedly different, particularly in view of the relatively modest differences between the late-type stellar populations of Fornax and the more luminous massive systems in Table 4. (Recall that Fornax also possesses five globular clusters.) In fact, aside from the diffuse nature of Sculptor, there are as yet no detected significant differences between its stellar popula- tion and that of $\omega$ Cen. ${ }^{6}$ What, then can account for the difference between Sculptor and Fornax? It is generally assumed, with the exception of $\omega$ Cen, that the reason that no globular cluster has a measurable range in heavy metal abundance is that the stars in each cluster formed over a very short time interval with self-enrichment suppressed either because star formation was extremely efficient with no leftover gas to form succeeding generations of stars, or because, once the first generation formed in a cluster, for one reason or another, the cluster became incapable of retaining any remaining gas. Even in $\omega \mathrm{Cen}$, the fact that the horizontal branch is populated almost exclusively on the blue side strictly limits the time span over which the enrichment process could have taken place. Thus, we suggest that the fundamental difference between Fornax and Sculptor is that Sculptor was rapidly and almost completely depleted of its nonstellar mass content shortly after the initial generations of stars formed. Varying times of gas retention in dwarf galaxies have also been advanced by AM as the explanation for Fornax's C star population. What we are emphasizing here, though, is the abrupt transition in the characteristics of the cool stellar population that is so obviously encountered in passing from Fornax to Sculptor as compared to the similar cool stellar populations of those systems at least as massive as Fornax for which we have good data. The origin of such a discontinuity is not apparent in most schemes for massdependent depletion rates of interstellar material from galaxies, e.g., supernova-driven galactic winds as discussed by Larson (1974). One speculative possibility is that Sculptor had a fairly dramatic encounter with the Milky Way (or perhaps with the Magellanic Clouds) early on in its history that stripped it of its gas, thus inhibiting subsequent star formation.

We thank F. Schweizer for providing us with his direct plate of Sculptor and A. Dressler for a print of his Fornax plate. J. A. F. acknowledges an informative conversation with Gary Da Costa and thanks Harvey Richer for a preprint of the Demers and Kunkel paper. We appreciate the useful comments made by a number of our colleagues at CITO on an earlier draft of this paper. J. Elias suggested several important additions to our initial work. J. G. C. is grateful for a grant from the Caltech Recycling Center. Infrared equipment at Las Campanas was built with funds provided in part by NSF grant AST 76-22676 to S. E. Persson.

\footnotetext{
${ }^{6}$ The difference between the horizontal branch populations of the two systems is not regarded as significant for the purposes of this discussion since, as we have just pointed out, an age difference of $1-2 \times 10^{9}$ yr could be sufficient to explain it.
}

\section{REFERENCES}

Aaronson, M., and Mould, J. 1980, Ap. J., 240, 904 (AM).

Baumert, J. H. 1974, Ap. J., 190, 85.

Blanco, V. M. 1965, in Galactic Structure, ed. A. Blaauw and M. Schmidt (Chicago: University of Chicago Press), p. 241.

Blanco, B. M., Blanco, V. M., and McCarthy, M. F. 1978, Nature, 271, 638.
Blanco, V. M., Hoag, A. A., and McCarthy, M. F. 1978, in IAU Symposium 80, The HR Diagram, ed. A. G. Davis, Philip and D. S. Hayes (Dordrecht: Reidel), p. 33.

Blanco, V. M., McCarthy, M. F., and Blanco, B. 1980, Ap. J., 242, 938 (BMB). 
Castellani, V., Giannone, P., and Renzini, A. 1973, in IAU Colloguium 21, Variable Stars in Globular Clusters and in Related Systems, ed. J. D. Fernie (Dordrecht: Reidel), p. 197.

Catchpole, R. M., and Feast, M. W. 1973, M.N.R.A.S., 164, 11P

Cohen, J. G., Frogel, J. A., and Persson, S. E. 1978, Ap. J., 222, 165.

Cohen, J. G., Frogel, J. A., Persson, S. E., and Elias, J. H. 1981, Ap. J., 249, 481 (CFPE).

Danziger, I. J. 1973, Ap. J., 181, 641

Demers, S., and Kunkel, W. E. 1979, Pub. A.S.P., 91, 761 (DK).

Demers, S., Kunkel, W. E., and Hardy, E. 1979, Ap. J., 232, 84

Feast, M. W., and Lloyd Evans, T. 1973, M.N.R.A.S., 164, 15P.

Frogel, J. A. 1981, in Physical Processes in Red Giants, ed. I. Iben, Jr. and A. Renzini (Dordrecht: Reidel), p. 63.

Frogel, J. A., and Cohen, J. G. 1981, Ap. J., 251, in press.

Frogel, J. A., Cohen, J. G., Persson, S. E., and Elias, J. H. 1981, in Physical Processes in Red Giants, ed. I. Iben, Jr. and A. Renzini (Dordrecht: Reidel), p. 159.

Frogel, J. A., Persson, S. E., Aaronson, M., and Matthews, K. 1978, Ap. J., 220, 75.

Frogel, J. A., Persson, S. E., and Cohen, J. G. 1980, Ap. J., 239, 495. 1981, Ap. J., 246, 842

Frogel, J. A., and Whitford, A. E. 1981, paper presented at the 157 th meeting of the AAS

Gingold, R. A. 1974, Ap. J., 193, 177.

Hodge, P. W. 1965, Ap. J., 142, 1390.

-. 1971, Ann. Rev. Astr. Ap., 9, 35

Iben, I., Jr. 1981, Ap. J., 243, 987.
Iben, I., Jr., and Truran, J. W. 1978, Ap, J, 220, 980.

Johnson, H. L. 1966, Ann. Rev. Astr. Ap., 4, 193.

Kron, G. E. 1974, private communication quoted in Peterson, C. J. and King, I. R. 1975, A.J., 80, 427.

Kunkel, W. E., and Demers, S. 1977, Ap. J., 214, 21 (KD).

Larson, R. B. 1974, M.N.R.A.S., 169, 229.

Mendoza, E. E., and Johnson, H. L. 1965, Ap. J., 141, 161.

Mould, J., and Aaronson, M. 1980, Ap. J., 240, 464.

Norris, J., and Bessell, M. S. 1978, Ap. J. (Letters), 225, L49.

Paczynski, B. 1971, Acta Astr., 21, 417.

Persson, S. E., Frogel, J. A., Cohen, J. G., Aaronson, M., and Matthews, K. 1980, Ap. J., 235, 452 .

Pilachowski, C. A., Canterna, R., and Wallerstein, G. 1980, Ap. J. (Letters), 235, L21.

Renzini, A. 1981, in Physical Processes in Red Giants, ed. I. Iben, Jr and A. Renzini, (Dordrecht: Reidel), p. 165.

Renzini, A., and Voli, M. 1981, Astr. Ap., 94, 175.

Richer, H. B. 1981, Ap. J., 243, 744.

Sackmann, I. -J., Smith, R. L., and Despain, K. H. 1974, Ap. J., 187, 555

Scalo, J. M., and Miller, G. E. 1979, Ap. J., 233, 596.

van Agt, S. L. Th. J. 1978, Pub. David Dunlap Obs., 3, 205.

van den Bergh, S. 1969, Ap. J. Suppl., 19, 145.

Whitford, A. E. 1978, Ap. J. 226, 777.

Whitford, A. E., and Rich, R. M. 1981, paper presented at the 157 th meeting of the AAS.

Wood, P. R. 1981, in Physical Processes in Red Giants, ed. I. Iben, Jr. and A. Renzini, (Dordrecht: Reidel), p. 135.

\section{M. Blanco and JAY A. Frogel: Cerro Tololo Inter-American Observatory, Casilla 603, La Serena, Chile}

Judith G. Cohen: Palomar Observatory, California Institute of Technology, Pasadena, CA 91125

M. F. MCCARTHY: Specola Vaticana, Vatican City I-00120 